\title{
RF plasma generation in the chamber with the conducting walls
}

\author{
Andey Gavrikov ${ }^{1,2}$, Sergey Kuzmichev ${ }^{1,2}$, Gennadiy Lizyakin ${ }^{2}$, Valentin Smirnov $^{2}$, Rinat Timirkhanov ${ }^{1,2}$, \\ Ravil Usmanov ${ }^{1,2}$ and Nazar Vorona, ${ }^{1,2}$, \\ ${ }^{1}$ Joint Institute for High Temperatures of the Russian Academy of Sciences (JIHT RAS), 125412, Moscow, Russia \\ ${ }^{2}$ Moscow Institute of Physics and Technology (MIPT), 141701, Dolgoprudny, Moscow Region, Russia
}

\begin{abstract}
This work is devoted to study of inductively coupled RF discharge within a metal chamber with a diameter of about $1 \mathrm{~m}$ and a length of about $2 \mathrm{~m}$ in argon gas (RF frequency is $5.28 \mathrm{MHz}$ ). The spatial distributions of electron temperature and density depending on the magnetic induction (from 0 to $0.2 \mathrm{~T}$ ), plasma-forming gas pressure $(0.1-10 \mathrm{mTorr})$ and RF power absorbed in the plasma are presented. For fixed gas pressure (6 mTorr) electron temperature decrease with increasing of magnetic field was found.
\end{abstract}

\section{Introduction}

Plasma separation method, proposed in [1] for spent nuclear fuel and radioactive wastes reprocessing, can to work in practice at about $1 \mathrm{~m}$ typical size of plasma volume, $0.1-0.2 \mathrm{~T}$ magnetic induction and about $1 \mathrm{kV}$ electrical potential. The presence of buffer plasma with magnetized electrons allows creation conditions for compensation of space charge [2] and reproduction of necessary electric field profile providing efficient spatial separation of ion flows. This circumstance removes a limitation on ion current value and allows obtaining the reprocessing productivity attractive from industrial point of view.

The paper is devoted to experiments with plasma generation in the presence of magnetic field in the volume limited by conductive walls. Conditions are observed where plasma separation method of elements can be realized (plasma density is about $10^{12}-$ $10^{13} \mathrm{~cm}^{-3}$, electron temperature is about units of $\mathrm{eV}$ ) [3]. The properties of RF plasma source observed. Also the results of the measurements of argon plasma parameters (electron temperature and density) generated by the RF source are presented.

\section{Plasma source: features of generation conditions and construction}

The buffer plasma source for the SNF plasma separation method should satisfy a number of conditions:

1. The density of a plasma forming gas should not exceed $10^{13} \mathrm{~cm}^{-3}$, otherwise collisions with separated ions become significant and their flows disintegrate and mix. On the other hand plasma density should be as high as possible, that increase productivity of the method.

2. Electron energy $T_{e}$ of buffer plasma should not exceed the second ionization potential of separated ions $(\leq 10 \mathrm{eV})$.
3. The buffer plasma should provide a reproduction of required profile of electrical potential for efficient separation process. The physical mechanism of creation of electrical potential in plasma with magnetized electrons can be described as follows: the electron mobility along magnetic field $\left(B_{0} \approx 0.1 \mathrm{~T}\right)$ is several orders of magnitude higher than across it, therefore if a magnetic line of force lean by its ends on two electrodes immersed in plasma and biased at the same electrical potentials then this potential will be reproduced in a plasma volume. Thus a buffer plasma source should take into account a necessity of additional electrodes placement, which provide a potential of electric field.

4. As one of the important purposes of this work is a creation of engineering and physical fundamentals for the technology of SNF plasma separation, a significant part of the chamber must be metallic. This requirement is dedicated by conditions of radiation loads, manufacturability and large dimensions of the operating volume (typical size is more than $1 \mathrm{~m}$ ).

According to the authors opinion, the most perspective is a helicon RF discharge [4], because it allows entering the energy into a plasma volume across a magnetic field, and at power up to $10 \mathrm{kV}$ it is possible to generate plasma with density of about $10^{13} \mathrm{~cm}^{-3}$ in a large volume of $1 \mathrm{~m}^{3}$. The possibility of practical use of the helicon discharge for plasma generation in close experimental conditions was demonstrated in papers [57].

Given the above configuration of plasma source for SNF plasma separation method was proposed and it is presented on the Fig. 1. The vacuum chamber 1 is a metallic cylinder with internal diameter of $86 \mathrm{~cm}$ and length of $220 \mathrm{~cm}$. The chamber 2 is hermetically separated from vacuum chamber 1 by dielectric wall passing RF radiation and by water-cooled Faraday shield. In the chamber 2 the antenna for RF plasma pumping is located. On the end faces of the chamber 1

Corresponding author: raraavis@ihed.ras.ru 
the electrodes able to provide electrical potentials into plasma are placed. The Helmholtz coils generate a magnetic field up to $0.2 \mathrm{~T}$ in the chamber 1. Operating frequency of RF generator is $5.28 \mathrm{MHz}$. A Rogowski coil is used for antenna current diagnostics. The power absorbed by a load is measured by directional couplers (sensors of incident and reflected waves).

Plasma density and electron temperature were measured by RF compensated double probe with cylindrical shape of the tips, each $0,35 \mathrm{~mm}$ in diameter and $8 \mathrm{~mm}$ long. Linear motion feedthrough is used to move the probe from the top of the plasma to the axis. The probe was located in the area between magnetic coils (Fig. 1).

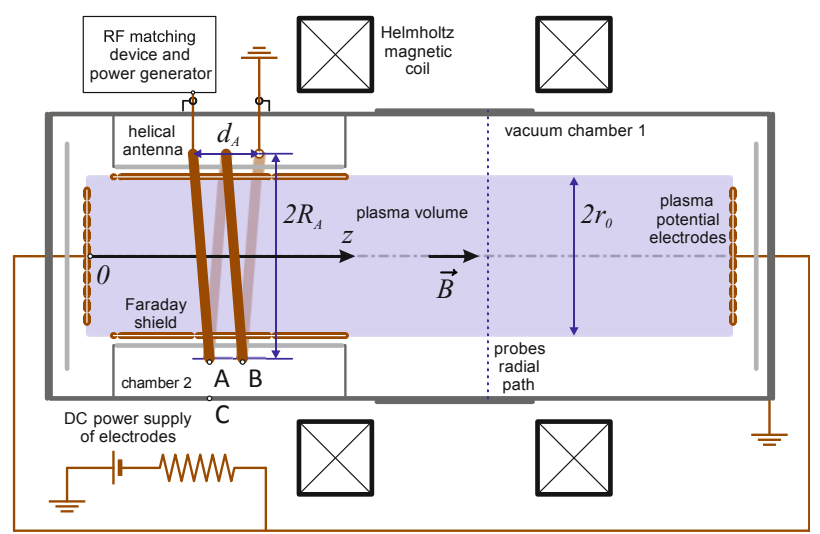

Fig. 1. Experimental setup layout and geometric parameters for the estimation of the plasma density.

For the estimation of antenna characteristics able to induce and maintain the helicon discharge with parameters required for plasma separation method (plasma density is up to $10^{13} \mathrm{~cm}^{-3}$, plasma volume is $\sim 1 \mathrm{~m}^{3}$, electron temperature is about units of $\mathrm{eV}$ ) an approach [8] was used. Estimations were performed in approximation of cold collision-free plasma with direct antenna placement within the single volume of gasdischarge chamber (GDC) (without chamber 2 and Faraday shield). All necessary parameters are shown in the Fig. 1.

In order the antenna radiation penetrates the argon plasma in homogenous magnetic field it is necessary for frequency $f$ to be in range of:

$f_{c, i}=\frac{e B_{0}}{2 \pi M_{A r}} \ll f \ll f_{c, e}=\frac{e B_{0}}{2 \pi m_{e}} \ll f_{p, e}=\frac{1}{2 \pi} \sqrt{\frac{n_{e} e^{2}}{m_{e} \varepsilon_{0}}}$, where $e$ is an elementary charge, $B_{0}$ is a magnetic field induction; $m_{e}$ and $M_{A r}$ are masses of electron and argon respectively; $n_{e}$ is an electron density $\left(\varepsilon_{0}-\right.$ vacuum permittivity). For $B_{0} \approx 0.1 \mathrm{~T}$ and $n_{e}=10^{13} \mathrm{~cm}^{-3}$ values of edge frequencies are $f_{c, i}=0.038 \mathrm{MHz}, f_{c, e}=$ $2830 \mathrm{MHz}, f_{p, e}=28.5 \mathrm{GHz}$. Chosen by the technical considerations operating frequency $f=5.28 \mathrm{MHz}$ belongs to an obtained range.

For helicon waves with the frequency $\omega$ and wave vector $\overrightarrow{\boldsymbol{k}}\left(k_{r}, k_{z}\right)$ spreading in unbound plasma with electron density $n_{e}$ at an angle of $\theta$ with respect to a magnetic field direction the dispersion relation is

$$
\begin{gathered}
\frac{k^{2}}{\omega^{2} / c^{2}}=\frac{\omega_{p e}^{2}}{\omega\left(\omega_{c e} \cos \theta-\omega\right)^{\prime}}, \\
k^{2}=k_{z}^{2}+k_{r}^{2}, \quad k_{z}=k \cdot \cos \theta,
\end{gathered}
$$

and waves are described by the expression like

$$
E, B \sim \exp j\left(\omega t-k_{z} z-m \varphi\right) \text {, }
$$

where $\varphi$ is an azimuthal angle, $m$ is an azimuthal number $(m=0, \pm 1, \pm 2, \ldots)$ and $c$ is a speed of light in vacuum.

For the helicon wave with $m=0$ spreading in plasma cylinder with radius $r_{0}$ minimal value of radial component $k_{r}$ of vector $\overrightarrow{\boldsymbol{k}}$ is $k_{r, \text { min }}=3,83 / r_{0}$ [8].

The projection of vector $k_{z}$ on the direction of vector $\vec{B}_{0}$ of magnetic induction is connected with the length of antenna $d_{A}$ (Fig. 1) as follows:

$$
k_{z}=\pi(2 l+1) / d_{A}, l=0,1,2,3, \ldots .
$$

For electron density $n_{e}$ in a cylindrical area with radius $r_{0}=25 \mathrm{~cm}$ corresponding to the helicon wave with frequency of $f=5.28 \mathrm{MHz}$ and to the lowest value of wave vector induced by the antenna with length of $d_{A}=0.2 \mathrm{~m}$ we have $\left(\mu_{0}-\right.$ vacuum permeability):

$$
n_{e}=\frac{k_{\min } k_{z, \min } B_{0}}{e \mu_{0} \cdot 2 \pi f} \approx 5 \cdot 10^{12} \mathrm{~cm}^{-3} .
$$

Thus such antenna can be used for a plasma source for realization of SNF plasma separation method.

Required power for RF pumping can be estimated from the relation [4]:

$$
P \approx 3500 \cdot\left(\frac{r_{0}^{2}}{d_{A}}\right) \cdot \frac{n_{e}\left[\mathrm{~cm}^{-3}\right]}{10^{13}\left[\mathrm{~cm}^{-3}\right]}[\mathrm{W}] .
$$

That gives $P \approx 55 \mathrm{~kW}$ for plasma density $n_{e}=$ $10^{12} \mathrm{~cm}^{-3}$.

Let us analyze electric field distribution occurring in the GDC before the breakdown and plasma formation (Fig. 1). In this case there are three components of electric field can be specified:

1. Electric field occurring due to the potential difference between antenna and GDC wall (points $A$ and $C$ Fig. 2). The magnitude of field $E_{1 \text {, max }}$ can be estimated as a ratio of antenna voltage $U_{\text {ant }}$ to a distance $h$ between antenna and GDC wall. For $h \approx 0.16 \mathrm{~m}$ we have $E_{\text {ant-wall }} \approx U_{\text {ant }} / h \approx 7 U_{\text {ant }}$.

2. Electric field occurring due to the potential difference between nearby antenna turns (points $A$ and $B$ Fig. 2). In this case the magnitude of field $E_{2, \max }$ can be estimated as a ratio of potential differences between the closest points on different antenna turns (in our case this value is about $U_{\text {ant }} / 2$ ) to a distance between turns. For $d=0.1 \mathrm{~m}$ we have $E_{\text {turn }} \approx U_{\text {ant }} / 2 d \approx 5 U_{\text {ant }}$.

3 . Vortex electric field magnitude near the antenna is estimated as $E_{\text {vort }} \approx U_{\text {ant }} / 2 \pi R_{A}$. For $R_{A}=0.3 \mathrm{~m}$ we have $E_{\text {vort }} \approx 0.5 U_{\text {ant }}$.

Given analysis shows that in the case of direct antenna placement within the single volume of GDC filed intensities in the central part of GDC will be more than an order of magnitude lower than field intensities near walls. Therefore the initial breakdown and emerging discharge plasma as well will be concentrated near the walls and the main part of the operating volume, where plasma presence is necessary, will be free of it. This conclusion is also proved by results of preliminary experiments (Fig. 2). 


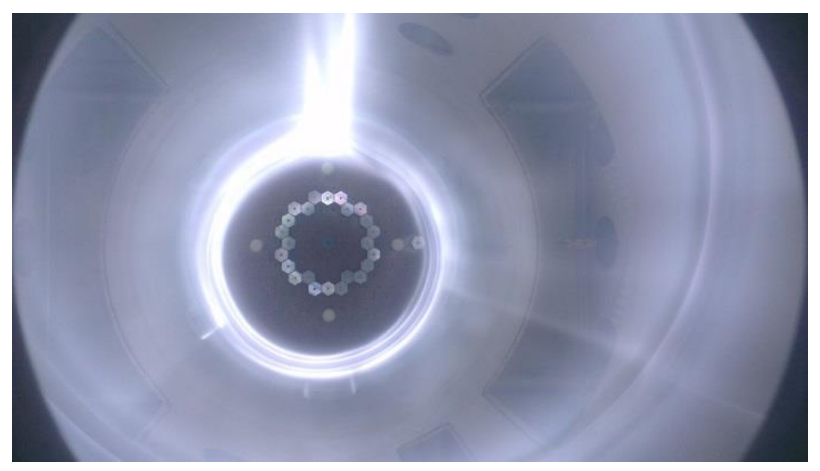

Fig. 2. Typicall discharge with direct antenna placement within the single volume (without chamber 2 and Faraday shield) $\left(P \approx 1.5 \mathrm{~kW}, p=9 \mathrm{mTorr}(\mathrm{Ar}), B_{0}=0.14 \mathrm{~T}\right)$.

To solve the problem the following actions were taken. Firstly, the antenna was placed in a separate volume hermetically isolated by dielectric divider (chamber 2). This volume is filled by air at atmospheric pressure that provides better electrical resistivity than in the main part of the chamber [9] and prevents a breakdown development between the antenna and the chamber. Secondly the Faraday shield made of conductive slits parallel to the magnetic lines was used [10-12]. The presence of the shield minimizes a capacitive coupling between the antenna and the plasma and increase the proportion of power transferred to the plasma through the inductive channel [13]. For inductive plasma a higher and more homogeneous electron density at relatively low electron temperature is typical [14] in contrast with capacitive plasma. Besides that inductive decoupling of plasma volume and antennas tract leads to a stabilization of the discharge and impedance of antennas tract as well [15] that allows efficiently calculate and perform adjusting independently of discharge parameters. It should be noted that Faraday shield also partially protects the dielectric divider surface, facing the plasma, from ion bombardment.

\section{The experiment}

With the help of RF plasma sources presented in previous section, plasma generation in different conditions were carried out.

Argon was used as a plasma forming gas and its pressure was in range of 2-6 mTorr. Stable operation of RF discharge was obtained at magnetic induction of $0-0.12 \mathrm{~T}$. The photo of typical obtained discharge is shown on the Fig. 3.

On the Fig. 4(a) the obtained distributions of electron temperature $T_{e}$ and density $n_{e}$ at magnetic induction $B_{0}=0.026 \mathrm{~T}$ and the buffer gas pressure $p=$ 5.9 mTorr are shown. Electron temperature in this regime changes non monotonically and has a local maximum at $r=5 \mathrm{~cm}$ of from the discharge axis where its value reaches $\sim 9 \mathrm{eV}$. Closer to the chamber periphery $T_{e}$ decreases to the value of $4 \mathrm{eV}$. Electron density $n_{e}$ is also distributed non monotonically on radius and has a fall in the region of $T_{e}$ peak, where it is equal to 1.7 . $10^{11} \mathrm{~cm}^{-3}$. On the discharge axis a density oscillations were observed that probably connected with instability of RF discharge at specified experiment parameters.

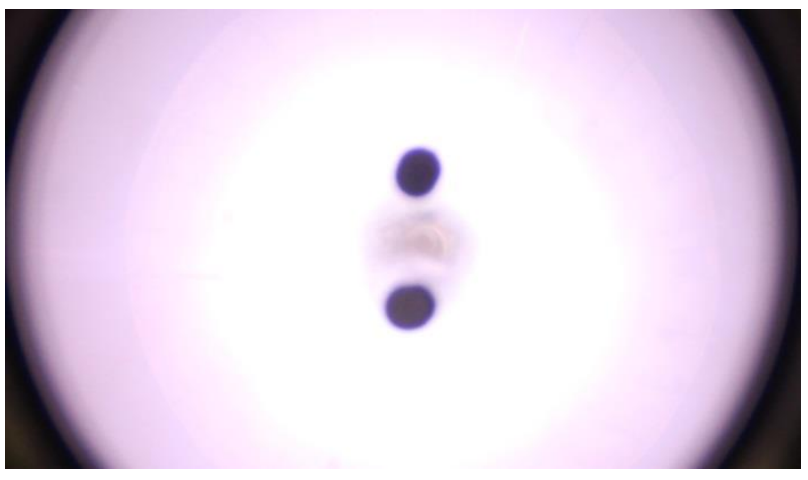

Fig. 3. Typicall discharge with Faraday shield $(P \approx 1 \mathrm{~kW}$, $p=5.9$ mTorr (Ar), $B_{0}=0.026 \mathrm{~T}$. Spots in the center are the diagnostic elements in front of the glass).
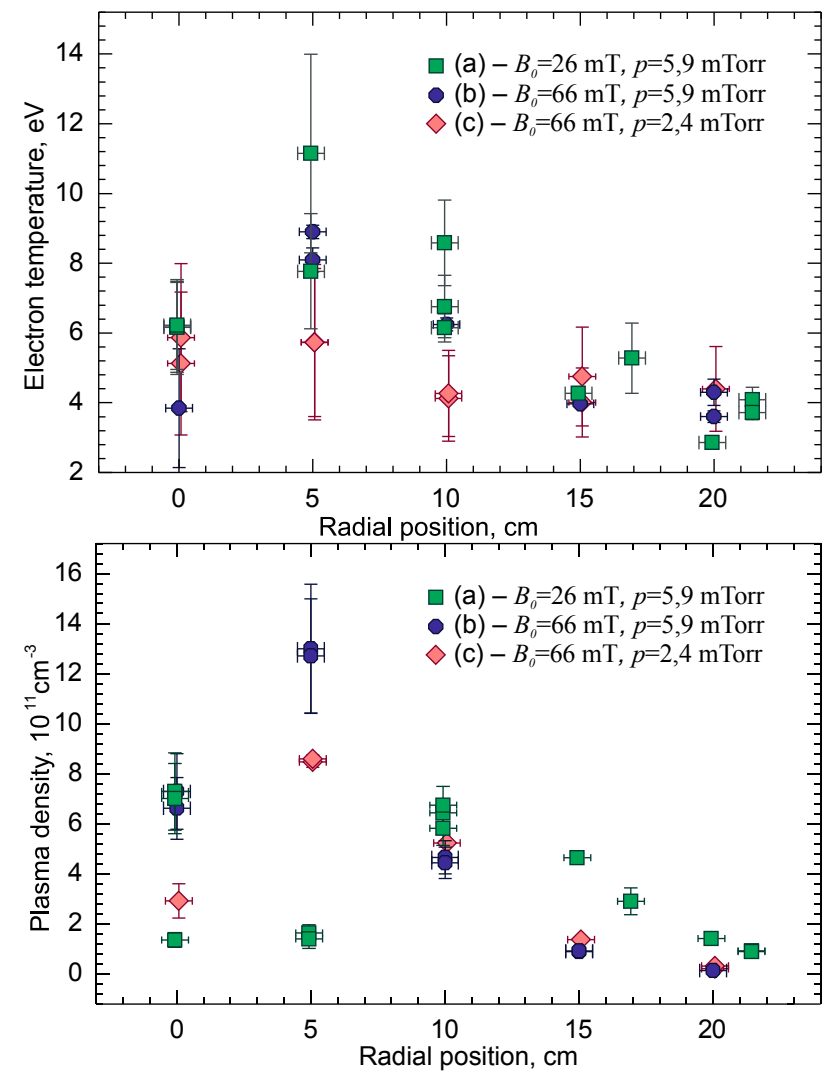

Fig. 4. Plasma density and electron temperature profiles of RF discharge at mentioned parameters (a-c) and $P \approx 1 \mathrm{~kW}$.

Electron temperature and density profiles were also measured at higher magnetic induction $B_{0}=0.066 \mathrm{~T}$ and the same argon pressure $p=5.9$ mTorr (Fig. 4(b)). The graphs show that increase of magnetic field causes a smoothing of electron temperature profile. The average value of $T_{e}$ in this regime decreases from the center to the periphery from 6 to $4 \mathrm{eV}$. Radial profile of electron density has a local maximum of $1.2 \cdot 10^{12} \mathrm{~cm}^{-3}$ at $r=5 \mathrm{~cm}$ from the discharge axis and density decreases farther on radius. At $r=20 \mathrm{~cm}$ from the axis density falls to a value of about $1.2 \cdot 10^{10} \mathrm{~cm}^{-3}$.

Electron temperature and density profiles at the same magnetic field $B_{0}=0.066 \mathrm{~T}$ but at a lower argon pressure $p=2.4$ mTorr are presented on the Fig. 4(c). 
The decrease of the buffer gas pressure in contrast with previous regime leads to a non monotonically distribution of electron temperature along the radius of the chamber. The temperature has a local maximum of $\sim 9 \mathrm{eV}$ at $r=5 \mathrm{~cm}$ from the discharge axis. At the remaining points it stays in range of $4-6 \mathrm{eV}$. The shape of plasma density distribution is not changed but the ratio of maximum density at $r=5 \mathrm{~cm}$ to a density on the axis is altered. If at the argon pressure of 5.9 mTorr the ratio is 2 then at the pressure of 2.4 mTorr it already becomes 3 .
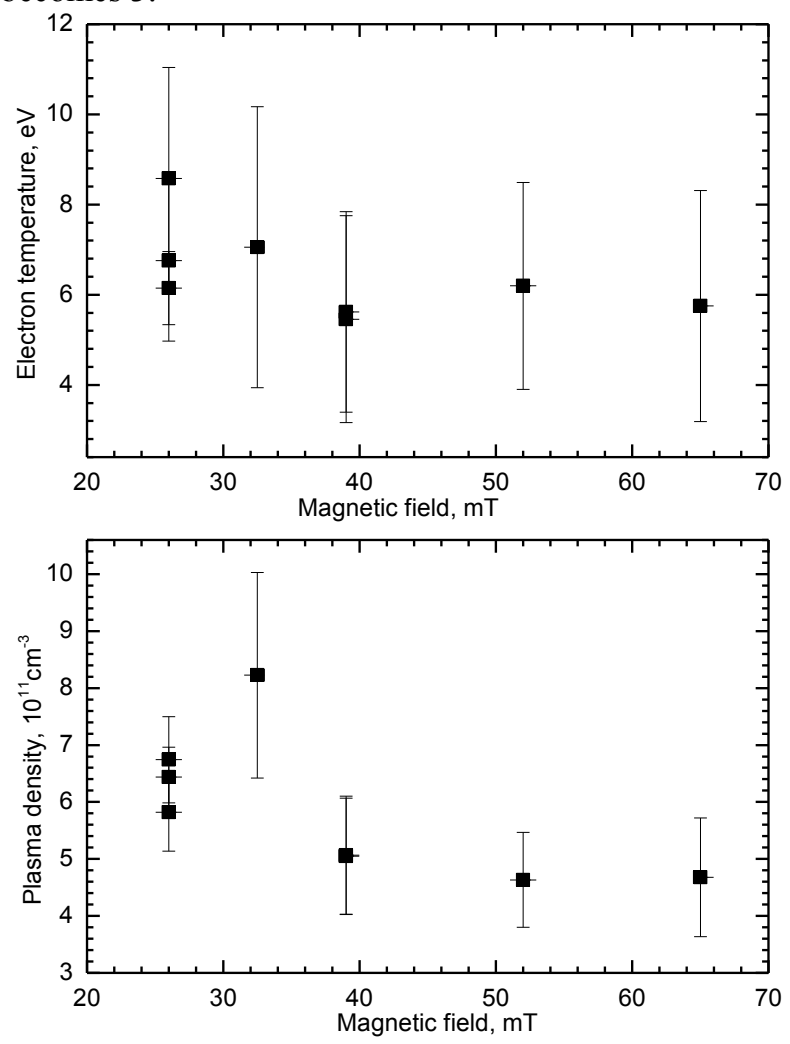

Fig. 5. The dependencies of electron temperature and density on magnetic field in the center of the chamber at the buffer gas pressure $p=5.9$ mTorr.

In presence of RF plasma electrical potential on the chamber axis depending on magnetic field was measured by the method of floating probe [16]. Argon pressure was 6 mTorr. The measurement results are shown on the Fig. 6.

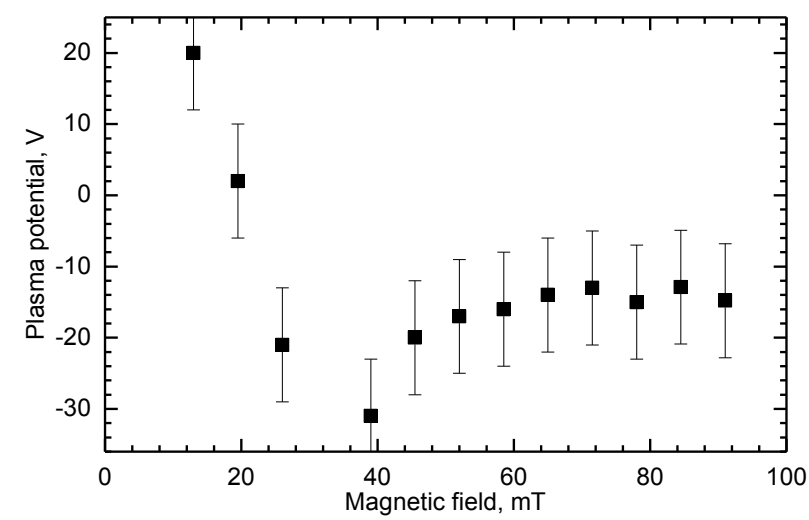

Fig. 6. Plasma potential versus magnetic field on the axis of the chamber.
Obtained relation demonstrating the transition of electrical potential through zero point can be explained in the following way. With the increase of magnetic field from 0 to $0.02 \mathrm{~T}$ electrons become more magnetized and accumulate on the axis, and ions recombine mainly on the wall, due to this processes the potential on the axis take negative values. Further increase of magnetic induction leads to partial ion magnetization and electrical potential becomes more compensated.

The study was supported by a grant of the Russian Science Foundation (project No. 14-29-00231).

\section{References}

1. V. P. Smirnov, A. A. Samokhin, N. A. Vorona et al. Plasma Phys. Rep., 39, 456 (2013)

2. A. I. Morozov, Introduction to Plasma Dynamics (CRC Press, 2012)

3. N. A. Vorona, A. V. Gavrikov, A. A. Samokhin et al., Phys. Atom. Nuclei, 78, 1624 (2015)

4. F. F. Chen, in High Density Plasma Sources, edited by O. A. Popov (Noyes, New York, 1995)

5. R. Freeman, S. Agnew, F. Anderegg et al., AIP Conf. Proc, 694, 403 (2003)

6. C. E. Ahfeld, J. D. Wagoner, D. L. Sevier et al., Fusion Engineering 2005, Twenty-First IEEE/NPS Symposium, 1 (2005)

7. A. Litvak, S. Agnew, F. Anderegg, B. Cluggish et al., 30th EPS Conf. Contr. Fusion and Plasma Phys, 27A, O-1.6A (2003)

8. P. Chabert, N. Braithwaite, Physics of RadioFrequency Plasmas (Cambridge University Press, 2011)

9. V. A. Lisovskiy, V. D. Yegorenkov, J. Phys. D: Appl. Phys., 31, 3349 (1998)

10. B. P. Cluggish, F. A. Anderegg, R. L. Freeman et. al., Phys. Plasmas, 12, 057101 (2005)

11. P. Y. Nabhiraj, Ranjini Menon, G. Mohan Rao et. al, Nucl. Instrum. Methods Phys. Res. A, 621, 57 (2010)

12. B. W. Longmier, A. D. Gallimore, N. Hershkowitz, Plasma Sources Sci. Technol., 21, 015007 (2012)

13. E. A. Kralkina, P. A. Nekliudova, V. B. Pavlov et al., Plasma Sources Sci. Technol., 26, 055006 (2017)

14. E. A. Kral'kina, Phys. Usp., 51, 493 (2008)

15. M. H. Khater, L. J. Overzet, J. Vac. Sci. Technol. A, 19, 785 (2001)

16. F. F. Chen, Lecture Notes on Principles of Plasma Processing (Plenum/Kluwer Publishers, 2002) 